\title{
RUNX3 modulates hypoxia-induced endothelial-to-mesenchymal transition of human cardiac microvascular endothelial cells
}

\author{
YANHUA LIU $^{1 *}$, JIN ZOU $^{1 *}$, BINGONG LI $^{1}$, YUQIN WANG $^{2}$, DELONG WANG $^{2}$, \\ YANQIN HAO ${ }^{1}, \mathrm{XUAN} \mathrm{KE}^{1}$ and XINGXING LI ${ }^{1}$ \\ ${ }^{1}$ Department of Cardiology, The First Affiliated Hospital, Nanchang University, Nanchang, Jiangxi 330006; \\ ${ }^{2}$ Department of Cardiology, Loudi Central Hospital, Loudi, Hunan 417000, P.R. China
}

Received May 21, 2016; Accepted May 17, 2017

DOI: $10.3892 /$ ijmm.2017.2998

\begin{abstract}
Endothelial-mesenchymal transition (EndMT) is an essential mechanism in the cardiovascular system, for both cardiovascular development and cardiovascular diseases (CVDs). Recent studies indicate that runt-related transcription factor 3 (RUNX3) contributes to EndMT and endothelial cell dysfunction. However, the underlying molecular mechanism remains unknown. The present study was designed to investigate the role of RUNX3 in EndMT and endothelial cell function, and to elucidate the underlying molecular mechanism. Human cardiac microvascular endothelial cells (HCMECs) were incubated in strictly controlled hypoxic conditions $\left(1 \% \mathrm{O}_{2}\right)$. HCMECs were cultured under normoxic conditions $\left(21 \% \mathrm{O}_{2}\right)$, and then moved to a strictly controlled hypoxic environment $\left(1 \% \mathrm{O}_{2}\right)$. Under this hypoxic condition, the cells were transfected with the lentiviral vector containing RUNX3 or an empty lentiviral vector for $8 \mathrm{~h}$. After the cells were cultured under hypoxic conditions for 4 days, CD31 and $\alpha$-smooth muscle actin colocalization were assessed by immunofluorescence microscopy. Transwell migration and tube formation assays were used to examine the migration and angiogenesis ability. RT-qPCR and western blotting were used to determine the expression of molecules involved in EndMT. Hypoxia induced the transition of HCMECs to mesenchymal cells and markedly promoted tube formation and cell migration. Transforming growth factor- $\beta$ (TGF- $\beta$ ) and Notch signaling were activated during the hypoxia-induced EndMT of HCMECs. RUNX3 knockdown attenuated EndMT of HCMECs, promoted angiogenic phenotype, and reduced endothelial cell migration. In conclusion, our results showed
\end{abstract}

Correspondence to: Dr Bingong Li, Department of Cardiology, The First Affiliated Hospital, Nanchang University, 17 Yongwaizheng Street, Nanchang, Jiangxi 330006, P.R. China

E-mail: libingong08@163.com

*Contributed equally

Key words: Notch, endothelial-mesenchymal transition, hypoxia, runt-related transcription factor 3 , transforming growth factor- $\beta$ that RUNX3 knockdown attenuated hypoxia-induced EndMT and reversed endothelial cell functions. RUNX3 is a common downstream target of TGF- $\beta$ and Notch signaling, and may be a novel therapeutic target for treating CVD mediated by EndMT.

\section{Introduction}

Due to rising prevalence, poor clinical outcome, and high health care costs, cardiovascular disease (CVD) is becoming a major public health concern and is the leading cause of death worldwide. According to the report on Cardiovascular Disease in China (2014), approximately 290 million individuals in China suffer from CVD each year, and CVD accounts for two in every five deaths (1). Clinical and experimental evidence suggests that the rarefaction of cardiac capillaries, infiltration of myofibroblasts, and the deposition of collagen and other extracellular matrix proteins contribute to the progression of cardiac fibrosis and heart failure, two common clinical outcomes of CVD (2-4). While exisiting therapies for CVD, such as pharmacological and invasive therapies, can relieve symptoms and attenuate disease progression, there is still an urgent need for novel therapies to effectively treat or even cure CVD.

Endothelial-mesenchymal transition (EndMT), one subgroup of cellular phenotypic switching, can induce transcription factors, which can affect gene expression and promote the loss of cell-cell adhesion and the change from endothelial morphology and physiology to a mesenchymal phenotype, a change characterized by low expression of endothelial markers such as CD31 (Pecam-1) and vascular endothelial (VE)-cadherin and increased expression of mesenchymal markers such as $\alpha$-smooth muscle actin ( $\alpha$-SMA) and fibroblast-specific protein (FSP)-1. EndMT has been proven to play a pivotal role in cardiovascular development and disease progression including cardiac fibrosis (2,5-9). Recent studies indicate that EndMT can be mediated by transforming growth factor- $\beta$ (TGF- $\beta$ )/bone morphogenetic protein (BMP), Notch and Wnt- $\beta$-catenin and hypoxia signaling pathways $(2,10-12)$. However, the mechanism remains unclear.

Runt-related transcription factor 3 (RUNX3), a member of the mammalian runt domain transcription factor family, is located on human chromosomes $1 \mathrm{p} 36.1$. Approximately a decade ago RUNX3 was claimed to be a tumor suppressor 
that inhibited the expression of vascular endothelial growth factor (VEGF) and reduced the angiogenesis, growth, and metastasis of human gastric cancer (13). RUNX3 is a direct target gene of Notch in human endothelial cells and a downstream effector of the TGF- $\beta$ signaling pathway, and plays a critical role in angiogenesis, cell migration and invasion $(14,15)$. Recent studies suggest that RUNX3 knockdown enhances endothelial progenitor cell (EPC) function and that miR-130a regulates EPC autophagy through RUNX3 $(16,17)$.

This study was designed to investigate the role of RUNX3 in EndMT as well as its signaling pathways, and to gain insight into the underlying molecular mechanisms so that novel therapeutic strategies can be ultimately developed for CVD.

\section{Materials and methods}

Cell culture and treatment. Human cardiac microvascular endothelial cells (HCMECs), purchased from ScienCell Research Laboratories (Carlsbad, CA, USA), were maintained in endothelial cell medium (ScienCell Research Laboratories) according to the manufacturer's instructions and were regularly examined under an inverted microscope (TS100; Nikon, Tokyo, Japan). After the cells reached $90 \%$ confluence, a subculture of cells was diluted to 40,000 cells $/ \mathrm{ml}$, and was seeded at 10,000 cells $/ \mathrm{cm}^{2}$ in a T-25 flask precoated with $5 \mu \mathrm{g}$ fibronectin (ScienCell Research Laboratories). A healthy culture displays cobblestone-shaped morphology, and non-granular cytoplasm while the cell number doubled two to three days in culture. HCMECs in this study were used between passages 3 and 6 .

HCMECs were seeded in a 6-well plate at a density of 10,000 cells $/ \mathrm{cm}^{2}$, before they were cultured in normoxic conditions and were grown to $30-35 \%$ confluency. For hypoxia-induced EndMT, HCMECs were incubated in strictly controlled hypoxic conditions $\left(1 \% \mathrm{O}_{2}\right)$ for 1,4 and 5 days, respectively.

HCMECs were cultured under normoxic conditions before they were divided into 4 groups: i) control group, HCMECs were cultured under normoxic conditions for 4 days without any treatment; ii) hypoxia group, HCMECs were cultured under strictly controlled hypoxic conditions $\left(1 \% \mathrm{O}_{2}\right)$ for 4 days; iii) RUNX3i group, HCMECs were transfected with the lentiviral vector containing RUNX3 (RUNX3-RNAi lentivirus; Genechem, Shanghai, China) for $8 \mathrm{~h}$ and cultured under strictly controlled hypoxic conditions $\left(1 \% \mathrm{O}_{2}\right)$ for 4 days; iv) green fluoresent protein (GFP) group, HCMECs were transfected with an empty lentiviral vector (Genechem) for $8 \mathrm{~h}$ and cultured under strictly controlled hypoxic conditions $\left(1 \% \mathrm{O}_{2}\right)$ for 4 days.

Double immunofluorescence staining. HCMECs were grown in 24-well culture plates. After the cells reached $70-80 \%$ confluence, they were washed with cold phosphate-buffered saline (PBS) 3 times and fixed in 4\% paraformaldehyde (both from Beijing Solarbio Science and Technology, Co., Ltd., Beijing, China) for $30 \mathrm{~min}$ before they were permeabilized with solution containing 0.1\% Trition X-100 (Beijing Solarbio Science and Technology, Co., Ltd.). After being blocked with $10 \%$ BSA for $1 \mathrm{~h}$ at room temperature, HCMECs were further incubated with two primary antibodies, monoclonal mouse anti-CD31 antibody (Cell Signaling Technology, Inc., Danvers, MA, USA) and monoclonal rabbit anti- $\alpha$-SMA antibody (Abcam, Cambridge, UK), at $4^{\circ} \mathrm{C}$ overnight. Twenty-four hours later, the cells were kept in a dark room for $1 \mathrm{~h}$ at room temperature and mixed with two secondary antibodies: CY3 red-conjugated goat anti-mouse IgG and FITC green-conjugated goat anti-rabbit IgG (both from Aspen Bio, Wuhan, China). After $100 \mu$ l DAPI (Aspen Bio) was added into each well, the mixture was incubated in the dark at room temperature for another $5 \mathrm{~min}$. In the negative control group, the primary antibody was replaced with non-immune IgG. The cells were visualized and mounted using phase contrast fluorescence microscopy (IX51; Olympus, Tokyo, Japan). All images were measured using Photoshop CS 8.0 software.

The lentivirus (Genechem) in this study expressed GFP, so HCMECs transduced with lentivirus were incubated with two secondary antibodies: CY3 red-conjugated goat anti-mouse (Aspen Bio) and DyLight 405 AffiniPure goat anti-rabbit IgG (H+L) (Abbkine, Inc., Redlands, CA, USA).

Silencing of RUNX3 with RUNX3-RNAi lentivirus. The transduction of HCMECs with the lentivirus was performed according to the manufacturer's instructions with some modifications. The preliminary study confirmed that the MOI of HCMECs was 20, and that polybrene $(5 \mu \mathrm{g} / \mathrm{ml})$ could improve the infection rate. The cells were seeded at $30-40 \%$ confluency in 6-well plates with HCMEC culture medium. Twelve hours later, the cells in each well were incubated with 20 MOI RUNX3-RNAi lentivirus or control lentivirus (for control group) for $8 \mathrm{~h}$. Lentiviral infection was confirmed by the visualization of enhanced GFP under a fluorescence microscope (IX51; Olympus). Then, the culture medium was replaced with fresh HCMEC supplemented medium and the mixture was incubated under strictly controlled hypoxic conditions $\left(1 \% \mathrm{O}_{2}\right)$ for an additional 4 days.

$R N A$ extraction and reverse transcription-quantitative $P C R(R T-q P C R)$. Total RNA was extracted from HCMECs using TRIzol ${ }^{\circledR}$ reagent (Life Technologies, Grand Island, NY, USA) and the extracted RNA was precipitated with isopropanol following standard procedures. First-strand complementary DNA (cDNA) was synthesized with a cDNA synthesis kit (Promega, Beijing, China) in $20 \mu \mathrm{l}$ reaction mixture containing $5 \mu \mathrm{g}$ total RNA, according to the manufacturer's instructions. The synthesized cDNA was used for quantitative PCR (qPCR). $\beta$-actin was used as endogenous control and the primer sequences (Generay Biotech Co, Ltd., Shanghai, China) are listed in Table I. The reaction volume for qPCR was $20 \mu \mathrm{l}$, containing $2 \mu \mathrm{l}$ cDNA. qPCR was performed on a 7500 Real-Time PCR system (Applied Biosystems, Carlsbad, CA, USA) using the GoScript ${ }^{\mathrm{TM}}$ SYBR-Green PCR kit (Promega) according to the manufacturer's instructions. Relative mRNA expression levels for the genes of interest were analyzed using the $2^{-\Delta \Delta C t}$ method as previously described (18).

Total protein extraction and western blotting. HCMECs were homogenized in RIPA lysis buffer that contained protease inhibitor PMSF (both from Beijing Solarbio Science and Technology, Co., Ltd.). After the mixture was centrifuged at $12,000 \times \mathrm{g}$ for $10 \mathrm{~min}$ at $4^{\circ} \mathrm{C}$, the supernatant was collected. Total protein quantity was determined using the BCA protein assay kit (Beyotime Institute of Biotechnology, Haimen, 
Table I. RT-PCR primer sequences.

\begin{tabular}{lll}
\hline Genes & \multicolumn{1}{c}{ Forward $\left(5^{\prime} \rightarrow 3^{\prime}\right)$} & \multicolumn{1}{c}{ Reverse $\left(5^{\prime} \rightarrow 3^{\prime}\right)$} \\
\hline$\beta$-actin & GCCATGTACGTAGCCATCCA & GAACCGCTCATTGCCGATAG \\
CD31 & CCCAGCCCAGGATTTCTTAT & ACCGCAGGATCATTTGAGTT \\
VE-cadherin & CCAATCTGACCTGAAAAAGC & CCACCGTTTTCCGTGTAATA \\
$\alpha-S M A$ & AAGCACAGAGAGCAAAAGAGGAAT & ATGTCGTCCCAGTTGGTGAT \\
FSP-1 & AACTAAAGGAGCTGCTGACCC & TGTTGCTGTCCAAGTTGCTC \\
Notch1 & CGCACAAGGTGTCTTCCAG & AGGATCAGTGGCGTCGTG \\
Hey1 & GGAGAGGCGCCGCTGTAGTTA & CAAGGGCGTGCGCGTCAAAGTA \\
Hes1 & ACAGGGGGTAAAGGCTACTTTG & CTGCTGCTGCTGCGTTT \\
TGF- $\beta 1$ & CACTCCCACTCCCTCTCTC & GTCCCCTGTGCCTTGATG \\
Smad2 & GGAGCAGAATACCGAAGGCA 3 & CTTGAGCAACGCACTGAAGG \\
Slug & ATTCCAGAAACGCCACCTCC & GCTATTGAACACCAAAATGCAGG \\
Snail & AGATGCATATTCGG CCCAC & CCTCATGTTTGTGCAGGAGA \\
RUNX3 & AATCGGAAGCCTAACTACAGCGAG & CCTTGGCCTCAGAGAGCTGG \\
\hline
\end{tabular}

$\alpha$-SMA, $\alpha$-smooth muscle actin; FSP-1, fibroblast-specific protein-1; TGF- $\beta 1$, transforming growth factor- $\beta 1$; RUNX3, runt-related transcription factor 3 .

Table II. Primary antibodies.

\begin{tabular}{llll}
\hline Primary antibodies (Cat. no.) & \multicolumn{1}{c}{ Companies } & Dilute solution & Dilution rate \\
\hline Rabbit anti-GAPDH (ab181602) & Abcam & $5 \%$ non-fat milk & $1: 10,000$ \\
Mouse anti-CD31 (ab24590) & Abcam & $5 \%$ non-fat milk & $1: 500$ \\
Rabbit anti-VE-cadherin (\#2500) & Cell Signaling Technology & $5 \%$ BSA & $1: 500$ \\
Rabbit anti- $\alpha$-SMA (ab32575) & Abcam & $5 \%$ non-fat milk & $1: 5,000$ \\
Rabbit anti-FSP-1 (ab41532) & Abcam & $5 \%$ BSA & $1: 1,000$ \\
Rabbit anti-Notch1 (ab52627) & Abcam & $5 \%$ non-fat milk & $1: 1,000$ \\
Rabbit anti-Slug (\#9585) & Cell Signaling Technology & $5 \%$ BSA & $1: 1,000$ \\
Rabbit anti-Snail (\#3879) & Cell Signaling Technology & $5 \%$ BSA & $1: 1,000$ \\
Rabbit anti-Smad2/3 (bs-3484R) & Bioss & $5 \%$ non-fat milk & $1: 1,000$ \\
Rabbit anti-p-Smad2/3 (bs-8853R) & Bioss & $5 \%$ non-fat milk & $1: 1,000$ \\
Rabbit anti-RUNX3 (bs-0378R) & Bioss & $5 \%$ non-fat milk & $1: 1,000$ \\
Mouse anti-TGF- $\beta 2(a b 167655)$ & Abcam & $5 \%$ BSA & $1: 1,000$ \\
Rabbit anti-VEGFR1 (bs-0170R) & Bioss & $5 \%$ non-fat milk & $1: 1,000$
\end{tabular}

$\alpha$-SMA, $\alpha$-smooth muscle actin; FSP-1, fibroblast-specific protein-1; RUNX3, runt-related transcription factor 3 ; TGF- $\beta 2$, transforming growth factor- $\beta 2$; VEGFR1, vascular endothelial growth factor receptor 1 .

Table III. Secondary antibodies.

\begin{tabular}{ll}
\hline $\begin{array}{l}\text { Secondary antibodies } \\
\text { (Cat. no.) }\end{array}$ & \multicolumn{1}{c}{ Companies } \\
\hline $\begin{array}{l}\text { Goat anti-rabbit IgG } \\
\text { (ZB-2310) }\end{array}$ & $\begin{array}{l}\text { Zhongshan Golden Bridge } \\
\text { Biotechnology, Beijing, China } \\
\text { Goat anti-mouse IgG }\end{array}$ \\
(ZB-2305) & $\begin{array}{l}\text { Zhongshan Golden Bridge } \\
\text { Biotechnology, Beijing, China }\end{array}$ \\
\hline
\end{tabular}

China) according to the manufacturer's instructions. Protein samples were mixed with $6 \mathrm{X}$ loading buffer (Beijing Solarbio
Science and Technology, Co., Ltd.) and degenerated at $95^{\circ} \mathrm{C}$ for $10 \mathrm{~min}$. Forty micrograms of protein from each group was resolved on 4-12\% SDS-PAGE and then transferred onto polyvinylidene fluoride (PVDF) or nitrocellulose membranes (all from Beijing Solarbio Science and Technology, Co., Ltd.). The membranes were blocked with $10 \%$ non-fat milk in TBST for $1 \mathrm{~h}$, before they were incubated with the primary antibodies (Table II) at $4^{\circ} \mathrm{C}$ overnight. After being rinsed with TBST 3 times, the membranes were incubated with the secondary antibodies (Table III) for another hour for $1 \mathrm{~h}$ at room temperature in a shaker and the proteins were visualized using chemiluminescent kits (Beyotime Institute of Biotechnology). 

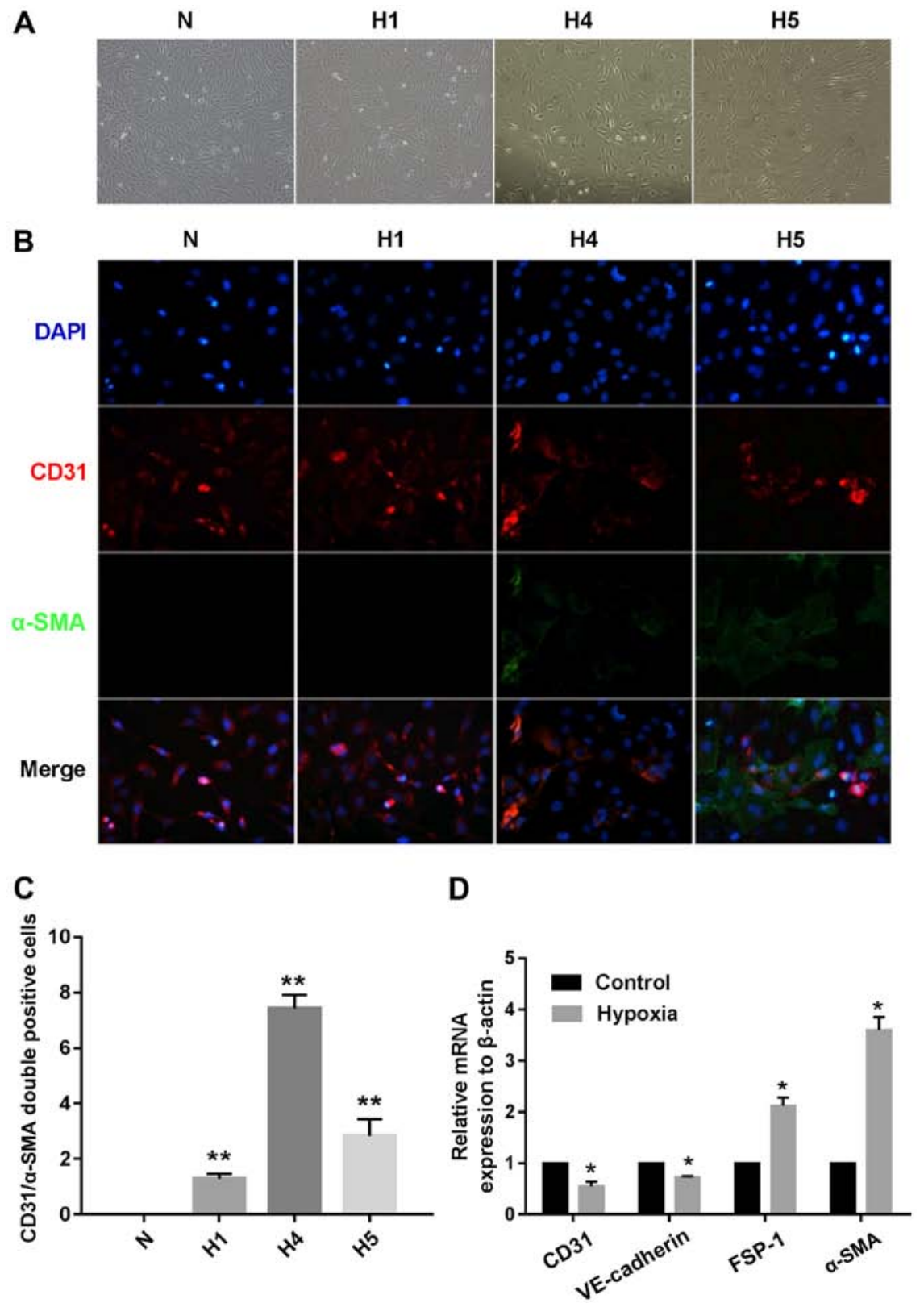

Figure 1. Hypoxia induces human cardiac microvascular endothelial cells (HCMECs) to mesenchymal transition. HCMECs were cultured under either normoxic conditions (N) or hypoxic conditions for 1, 4 and 5 days, respectively (H1, H4 and H5). (A) Representative phase contrast light microscopy images showing morphological changes in HCMECs (x100 original magnification). (B) Double immunofluorescence staining with antibodies to CD31 (red) and $\alpha$-smooth muscle actin ( $\alpha$-SMA) (green). Nuclei were counterstained with DAPI (blue). Acquisition of a spindle-shaped morphology upon hypoxia was correlated with $\alpha$-SMA acquisition and loss of CD31 (x200 original magnification). (C) Graph indicates quantification of CD $31^{+} / \alpha$-SMA ${ }^{+}$cells from each group. Bars indicate mean $\pm \mathrm{SD}, \mathrm{n}=7$ independent experiments. ${ }^{* *} \mathrm{P}<0.01$ vs. normoxia group. (D) RT-PCR data showing the mRNA expression levels of the endothelial markers (CD31 and VE-cadherin) and mesenchymal markers [fibroblast-specific protein (FSP)-1 and $\alpha$-SMA] in the normoxia and hypoxia groups. Results were normalized to that of reference gene $\beta$-actin ( $n=7$ independent experiments; ${ }^{*} \mathrm{P}<0.05$ vs. normoxia group).

Transwell migration assay. The migration assay was carried out in 24-well chambers of 6.5 -mm diameter with polycarbonate $8-\mu \mathrm{m}$ pore membrane filters (Transwell; Corning Life Sciences, Corning, NY, USA). HCMECs were resuspended in serum-free endothelial cell medium at $1 \times 10^{5}$ cells/ml. Two hundred microliters of cells were placed in the upper Transwell chambers and $500 \mu \mathrm{l}$ complete endothelial cell medium was added into each well in the lower 24-well plates. After incubation at $37^{\circ} \mathrm{C}$ for $24 \mathrm{~h}$, the migrated cells were stained with crystal violet (Aspen Bio). The images were obtained using an inverted microscope (IX51; Olympus) and the migrated cells were counted in 3 random fields (x200) for each well.
Tube formation assay. The neovascularization assays were performed in human fibrin matrices. In brief, the Matrigel (BD Biosciences, New Jersey, NY, USA) was thawed at $4^{\circ} \mathrm{C}$ overnight before being placed in a 96-cell plate $(50 \mu 1$ Matrigel/well). The cells were incubated at $37^{\circ} \mathrm{C}$ to allow solidification. Then, the cells from each group were harvested and resuspended $(200,000$ cells $/ \mathrm{ml})$, before they were seeded in plates $\left(50 \mu \mathrm{l} \mathrm{cells} /\right.$ well) and incubated at $37^{\circ} \mathrm{C}$ for $4-6 \mathrm{~h}$. The cell growth was examined under a phase-contrast microscope (IX51; Olympus). As described in the previous study (19), tube formation was the formation of a structure with its length four times longer than its width. The tube networks were 


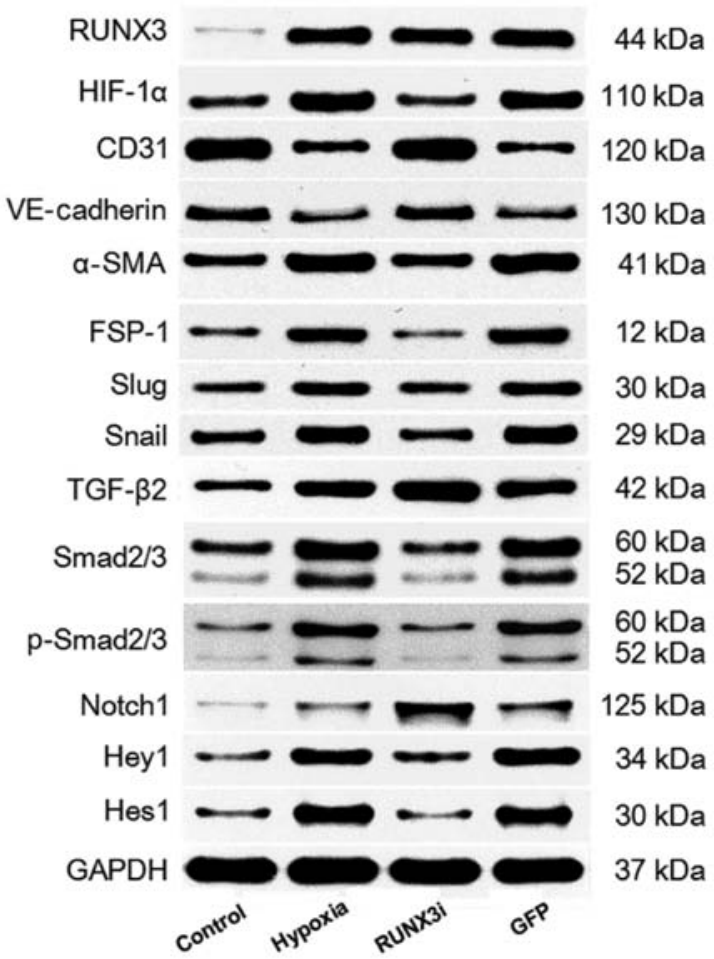

Figure 2. Western blotting of runt-related transcription factor 3 (RUNX3) and its targeting proteins in the control, hypoxia, RUNX3i and green fluoresent protein (GFP) groups.

photographed from 6 randomly chosen fields with a microscope. The results were analyzed using ImageJ software.

Statistical analysis. All data analysis was performed using GraphPad Prism 7.0 software (GraphPad Software Inc., San Diego, CA, USA). Experimental data are presented as mean \pm SD. Comparisons between groups were compared using one-way analysis of variance (ANOVA). P-value $<0.05$ was considered statistically significant.

\section{Results}

Hypoxia induces the transition of HCMECs to mesenchymal cells. To investigate the potential role of hypoxia in the induction of EndMT involving HCMECs, we incubated the HCMECs in strictly controlled hypoxic conditions $\left(1 \% \mathrm{O}_{2}\right)$ for 1,4 and 5 days, respectively. Under hypoxic conditions, the endothelial cells gradually developed an elongated spindle-shaped structure (Fig. 1A). The double immunofluorescence staining results showed that the hypoxia-treated HCMECs expressed proteins associated with fibroblasts ( $\alpha$-SMA), but lost proteins associated with endothelial cells (CD31). The changes became more obviously as cultivation time increased (Fig. 1B). $\mathrm{CD} 31^{+} / \alpha-\mathrm{SMA}^{+}$cells were visualized and assessed in both the normoxic HCMEC and hypoxic HCMEC groups, and the results showed that the number of $\mathrm{CD} 31^{+} / \alpha-\mathrm{SMA}^{+}$cells was significantly increased in the hypoxic HCMEC group at 4 days when compared to the others (Fig. 1C). These results demonstrated that hypoxia induced EndMT. Furthermore, the relative mRNA expression of endothelial and mesenthymal markers in both groups were assessed using RT-qPCR, and
A

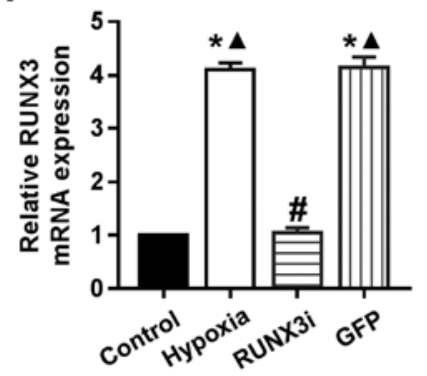

B
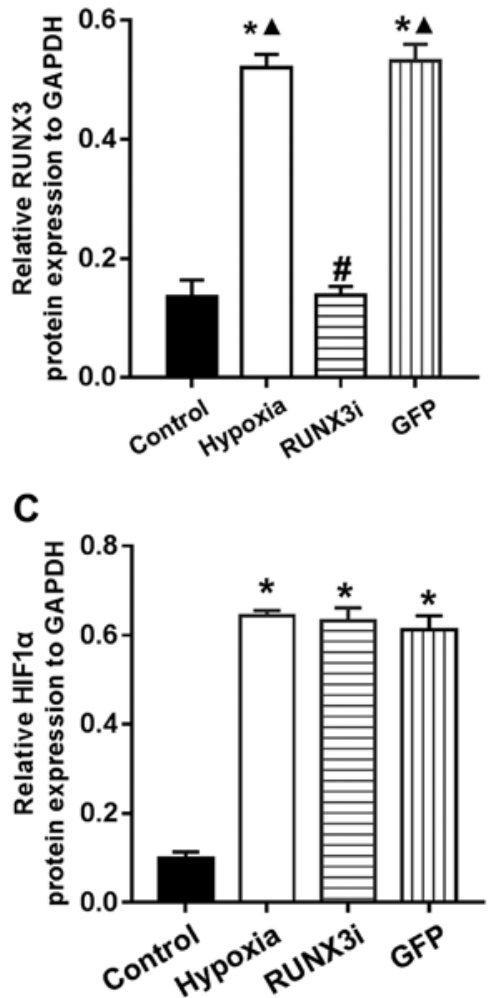

Figure 3. Hypoxia enhances runt-related transcription factor 3 (RUNX3) expression in human cardiac microvascular endothelial cells (HCMECs). (A) RT-PCR data showing the mRNA level of RUNX3 in the control, hypoxia, RUNX3i and green fluoresent protein (GFP) groups. Results were normalized to that of reference gene $\beta$-actin (bars indicate mean $\pm S D, n=7$; ${ }^{*} \mathrm{P}<0.05$ vs. control group; ${ }^{*} \mathrm{P}<0.05$ vs. hypoxia group; ${ }^{\wedge} \mathrm{P}<0.05$ vs. RUNX3i group). (B and C) Relative RUNX3 and hypoxia inducible factor-1 $\alpha$ (HIF-1 $\alpha)$ protein expression to GAPDH in the HCMECs from each group $\left(n=7,{ }^{*} \mathrm{P}<0.05\right.$ vs. control group; ${ }^{*} \mathrm{P}<0.05$ vs. hypoxia group; ${ }^{\wedge} \mathrm{P}<0.05$ vs. RUNX3i group).

the results showed that the endothelial markers, CD31 and VE-cadherin, were downregulated, while the mesenchymal markers, FSP-1 and $\alpha$-SMA, were upregulated in the hypoxic HCMEC group (Fig. 1D).

We examined the relative protein expression of RUNX3 and its targeting proteins by western blotting, and the expression blots are presented in Fig. 2.

Hypoxia enhances RUNX3 expression in HCMECs. To investigate the expression of RUNX3 in HCMECs during hypoxia-induced EndMT, we examined RUNX3 expression by RT-qPCR and western blotting. Both mRNA and protein levels of RUNX3 were low and increased significantly after the cells were cultured in hypoxic conditions 
A

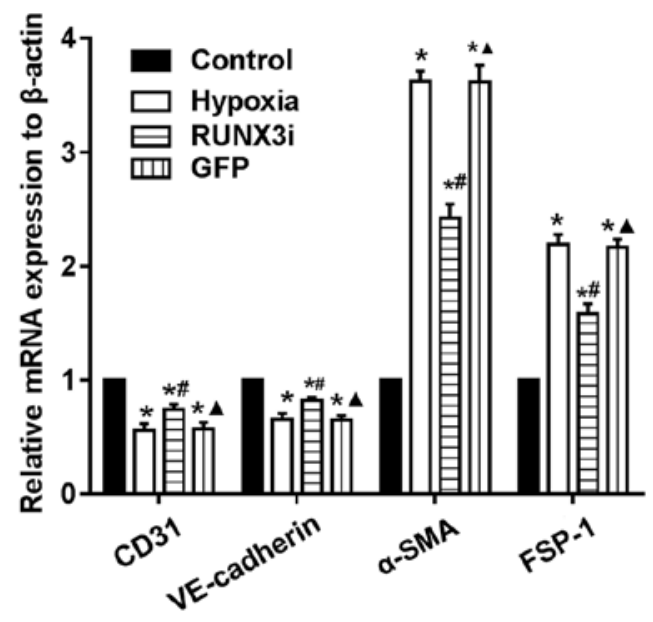

B

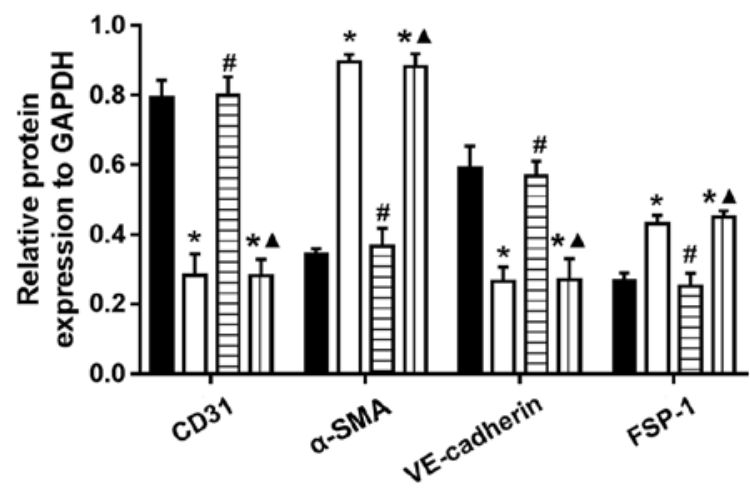

C

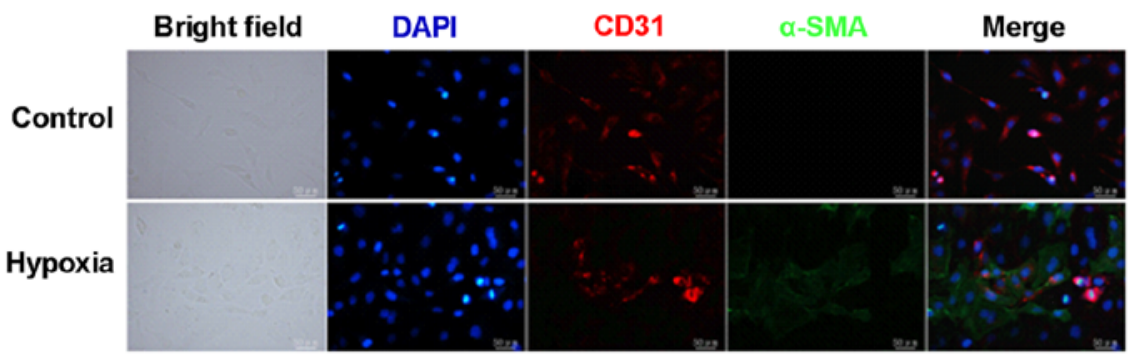

D

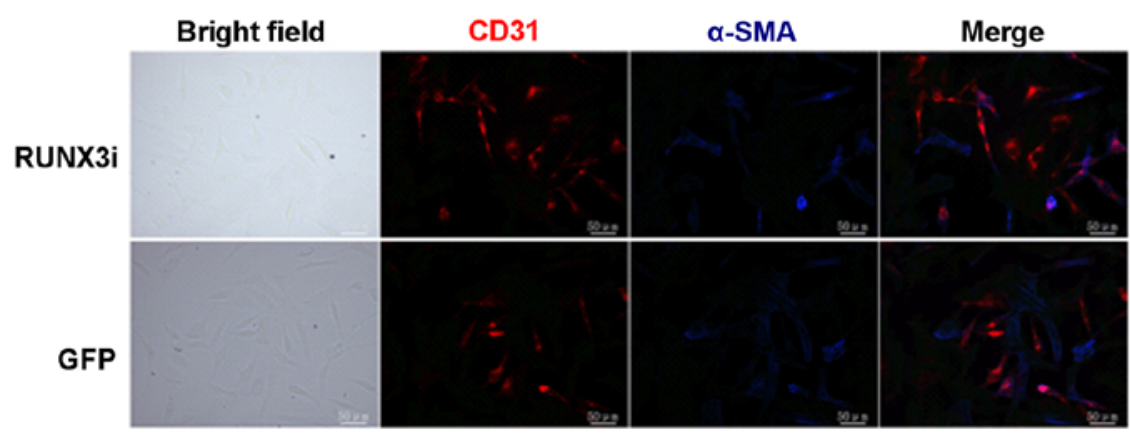

Figure 4. Knockdown of runt-related transcription factor 3 (RUNX3) attenuates endothelial-mesenchymal transition (EndMT) of human cardiac microvascular endothelial cells (HCMECs). (A) RT-PCR data showing the mRNA expression levels of the endothelial markers (CD31 and VE-cadherin) and mesenchymal markers [fibroblast-specific protein (FSP)- 1 and $\alpha$-smooth muscle actin ( $\alpha$-SMA)] in the HCMECs. Results were normalized to reference gene $\beta$-actin ( $\mathrm{n}=7,{ }^{*} \mathrm{P}<0.05$ vs. control group; ${ }^{\mathrm{P}} \mathrm{P}<0.05$ vs. hypoxia group; ${ }^{\wedge} \mathrm{P}<0.05$ vs. RUNX3i group). (B) Relative protein expression of CD31, VE-cadherin, FSP-1 and $\alpha$-SMA to GAPDH in the HCMECs from each group ( $\mathrm{n}=7,{ }^{*} \mathrm{P}<0.05$ vs. control group; ${ }^{*} \mathrm{P}<0.05$ vs. hypoxia group; ${ }^{\wedge} \mathrm{P}<0.05$ vs. RUNX3i group). (C) Double immunofluorescence staining with antibodies to CD31 (red) and $\alpha$-SMA (green). Nuclei were counterstained with DAPI (blue). The expression of CD31 was downregulated and $\alpha$-SMA upregulated in the hypoxia group compared with the control group. Scale bars, $50 \mu \mathrm{m}$. (D) Double immunofluorescence staining with antibodies to CD31 (red) and $\alpha$-SMA (blue). The expression of CD31 was upregulated and $\alpha$-SMA was downregulated in the RUNX3i group compared with the green fluoresent protein (GFP) group. Scale bars, $50 \mu \mathrm{m}$.

for 4 days (Figs. 2 and 3A and B). To examine the physical interaction between the transcriptional factor RUNX3 and EndMT, we knocked down the expression of RUNX3 in HCMECs using RUNX3-RNAi lentivirus. As shown in Fig. 3, the RUNX3-RNAi lentivirus decreased the expression of RUNX3 mRNA and protein to low levels, while the control lentivirus did not affect RUNX3 expression. The cultivation of HCMECs in hypoxic conditions induced intracellular accumulation of hypoxia-inducible factor- $1 \alpha$ (HIF- $1 \alpha$ ) (Figs. 2 and 3C). Therefore, our results showed that hypoxia enhanced RUNX3 expression in HCMECs.

Knockdown of RUNX3 attenuates EndMT of HCMECs. To examine whether RUNX3 plays a pivotal role in EndMT of
HCMECs, we suppressed RUNX3 expression in HCMECs with RUNX3-RNAi lentivirus and found that RUNX3 knockdown ameliorated hypoxia-induced EndMT (Fig. 4). Suppress of RUNX3 downregulated the mRNA expression of $\alpha$-SMA and FSP-1 and upregulated the expression of endothelial markers (Fig. 4A). Hypoxia induced a notable increase in the expression of $\alpha$-SMA and FSP-1, while RUNX3 knockdown attenuated $\alpha$-SMA and FSP-1 protein expression and increased CD31 and VE-cadherin levels in the HCMECs treated under a hypoxic condition (Figs. 2 and 4B). Double immunofluorescence staining assays were carried out to further confirm that knockdown of RUNX3 attenuated EndMT. CD31 was downregulated while $\alpha$-SMA was upregulated in the hypoxia group compared with the control 


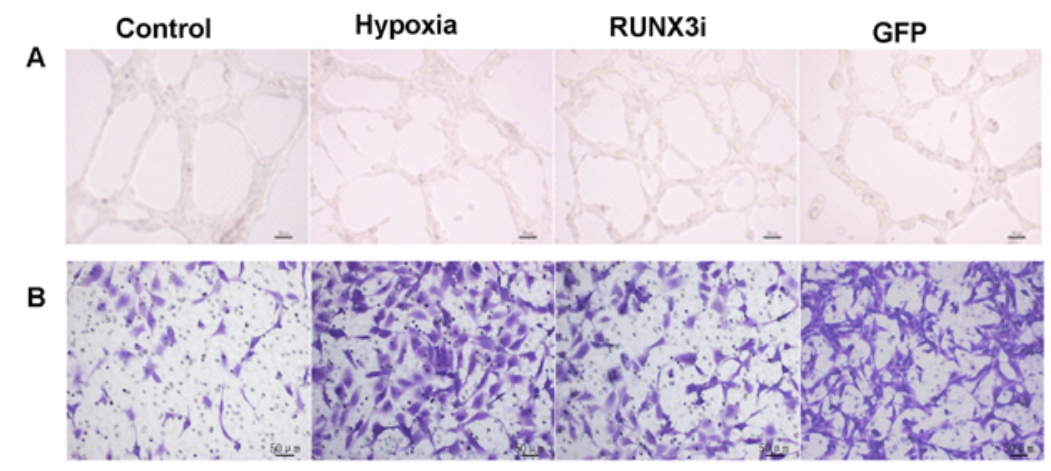

C
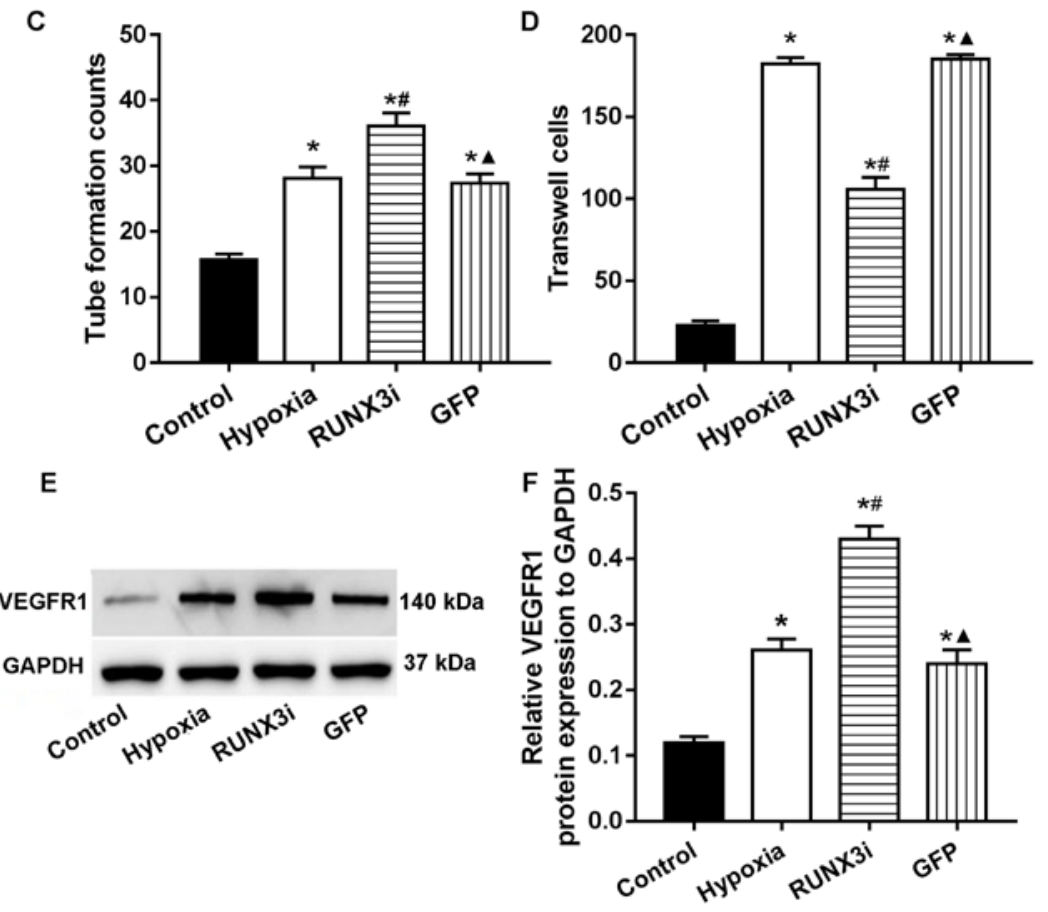

Figure 5. Low expression of runt-related transcription factor 3 (RUNX3) induces angiogenesis and decreases the migration of human cardiac microvascular endothelial cells (HCMECs). (A and B) Tube formation and Transwell migration assays (Scale bars, $50 \mu \mathrm{m}$ ). (C and D) Tube formation counts and the amounts of Transwell cells ( $\mathrm{n}=7,{ }^{*} \mathrm{P}<0.05$ vs. control group; ${ }^{*} \mathrm{P}<0.05$ vs. hypoxia group; ${ }^{\wedge} \mathrm{P}<0.05$ vs. RUNX3i group). (E) Western blotting of vascular endothelial growth factor receptor 1 (VEGFR1) in the HCMECs from each group. (F) Relative protein expression of VEGFR1 to GAPDH in the HCMECs from each group (n=7, ${ }^{*} \mathrm{P}<0.05$ vs. control group; ${ }^{*} \mathrm{P}<0.05$ vs. hypoxia group; ${ }^{\wedge} \mathrm{P}<0.05$ vs. RUNX3i group).

group (Fig. 4C), while CD31 was upregulated and $\alpha$-SMA was downregulated in the RUNX3i group compared with the GFP group (Fig. 4D). In brief, our data demonstrated that $\alpha$-SMA expression in HCMECs under hypoxia was inhibited by RUNX3-RNAi lentivirus, while the control lentivirus did not affect the protein expression.

RUNX3 modulates HCMEC function. In human gastric cancer cells, RUNX3 was found to suppress VEGF expression and reduce angiogenesis. Low RUNX3 expression was associated with increased VEGF expression and gastric cancer angiogenesis (13). To investigate the role of RUNX3 in HCMEC function, we examined the angiogenesis and migration ability via endothelial cell tube formation assay and Transwell migration assays. Our research showed that knockdown of RUNX3 adversely affected HCMEC function. Compared with that in the control HCMECs, hypoxia promoted tube formation and induced cell migration markedly, while knockdown of RUNX3 attenuated cell migration and increased angiogenesis in HCMECs treated under a hypoxic condition (Fig. 5A-D). These results indicated that the loss of RUNX3 positively affected angiogenic phenotype and reduced endothelial cell migration. As known, VEGF receptors in vascular endothelial cells are an important role during angiogenesis. In this study, we examined the expression of their proteins, and the results showed that the expression of VEGF receptor 1 (VEGFR1) in the cells from the hypoxia and RUNX3i groups were all increased, and the latter was more obvious (Fig. 5E and F).

RUNX3 regulates the expression of principal EndMT transcriptional factors. Several transcription factors induce EndMT. The principal EndMT transcriptional factors include zinc-finger binding transcription factors Snail and Slug $(12,20-22)$. As shown in Fig. 6, principal EndMT transcriptional factors Snail and Slug were upregulated under hypoxia, suggesting that hypoxia triggered the phenotypic changes of EndMT in HCMECs. Fu et al demonstrated that overexpression of RUNX3 induced EndMT in endothelial 
A

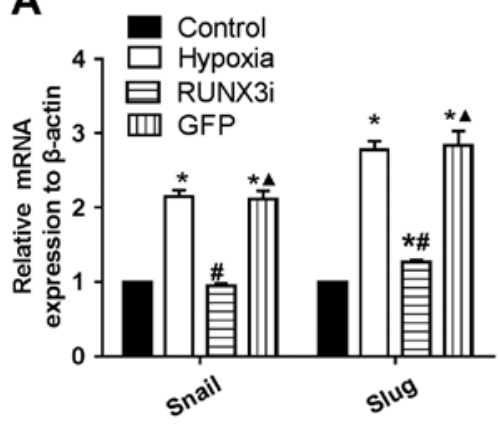

B

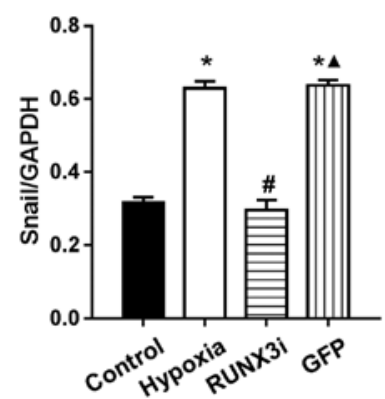

C

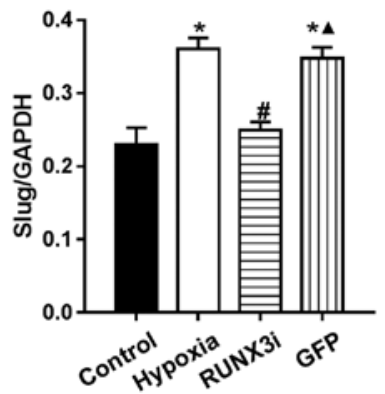

Figure 6. Runt-related transcription factor 3 (RUNX3) regulates expression of principal endothelial-mesenchymal transition (EndMT) transcriptional factors. (A) Gene expression of Snail and Slug by RT-PCR. Results were normalized to that of reference gene $\beta$-actin $\left(\mathrm{n}=7\right.$, ${ }^{*} \mathrm{P}<0.05$ vs. control group; ${ }^{\#} \mathrm{P}<0.05$ vs. hypoxia group; ${ }^{\wedge} \mathrm{P}<0.05$ vs. RUNX3i group). (B and C) Relative protein expression of Snail and Slug to GAPDH in the human cardiac microvascular endothelial cells (HCMECs) from each group ( $\mathrm{n}=7,{ }^{*} \mathrm{P}<0.05$ vs. control group; ${ }^{\#} \mathrm{P}<0.05$ vs. hypoxia group; ${ }^{\wedge} \mathrm{P}<0.05$ vs. RUNX3i group).

A

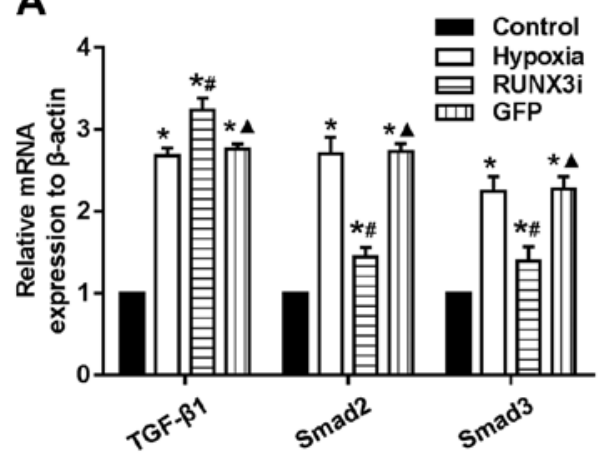

B

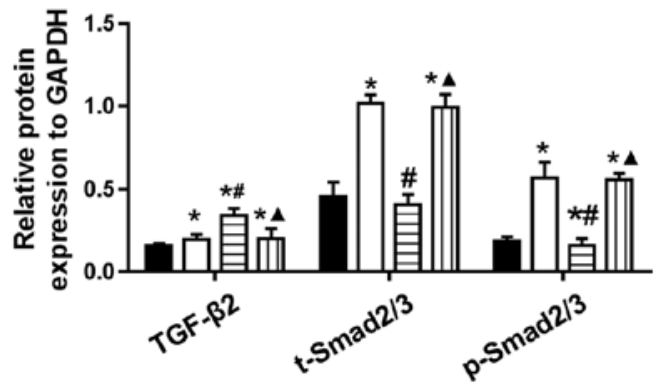

Figure 7. Changes in transforming growth factor- $\beta$ (TGF- $\beta$ ) signaling in hypoxia-induced endothelial-mesenchymal transition (EndMT). (A) mRNA levels of TGF- $\beta 1$, Smad 2 and Smad3 in human cardiac microvascular endothelial cells (HCMECs) were assessed by RT-PCR and normalized to that of reference gene $\beta$-actin ( $n=7,{ }^{*} \mathrm{P}<0.05$ vs. control group; ${ }^{*} \mathrm{P}<0.05$ vs. hypoxia group; ${ }^{\wedge} \mathrm{P}<0.05$ vs. RUNX3i group). (B) Relative protein level expression of TGF- $\beta 2$, Smad $2 / 3$ and p-Smad2/3 to GAPDH in the HCMECs from each group ( $\mathrm{n}=7,{ }^{*} \mathrm{P}<0.05$ vs. control group; ${ }^{*} \mathrm{P}<0.05$ vs. hypoxia group; ${ }^{\wedge} \mathrm{P}<0.05$ vs. RUNX3i group).

cells and upregulated the expression of Slug and Snail (14). To further investigate the interactions between these two positive regulators of EndMT and RUNX3, we found that the knockdown of RUNX3 in HCMECs upregulated the mRNA expression of endothelial markers and downregulated the expression of mesenchymal markers (Fig. 4A) such as Slug and Snail (Fig. 6A). The downregulation of Slug and Snail was further confirmed by western blotting (Figs. 2 and 6B and C). Thus, our data demonstrated that low expression of RUNX3 alleviated EndMT in HCMECs.

Changes in the TGF- $\beta$ signaling pathway in hypoxia-induced EndMT. Since hypoxia induces EndMT, we speculated that hypoxia may induce EndMT by activating TGF- $\beta$ signaling. We examined the expression of receptors and target genes involved in the signaling pathway, and found that hypoxia upregulated the mRNA expression of TGF- $\beta 1, \operatorname{Smad} 2$ and Smad3, while the knockdown of RUNX3 increased TGF- $\beta 1$ mRNA expression and reduced the mRNA expression of Smad2 and Smad3 (Fig. 7A). Upregulation of TGF- $\beta 2$, Smad $2 / 3$ and phosphorylation of Smad2/3 (p-Smad2/3) in HCMECs in the hypoxia group was further confirmed by western blotting and the levels of Smad2/3 and p-Smad2/3 in the HCMECs in the RUNX3i group were lower than those in the hypoxia group, while the level of TGF- $\beta 2$ was increased (Figs. 2 and 7B). Therefore, our data demonstrated that hypoxia-induced EndMT of HCMECs activated TGF- $\beta$ signaling and RUNX3 is a downstream target of TGF- $\beta$ signaling.

Changes in the Notch signaling pathway in hypoxia-induced EndMT. To examine whether hypoxia induces EndMT by activating Notch signaling through a positive feedback mechanism, we assessed the expression of Notch1, Hes1 and Hey1, and found that hypoxia enhanced Notch1, Hes1 and Heyl mRNA expression. Knockdown of RUNX3 markedly upregulated Notch-1 mRNA expression, while mRNA expression of Hes1 and Heyl was significantly downregulated (Fig. 8A). Similarly, changes in the protein levels in HCMECs in each group were further confirmed by western blotting (Figs. 2 and 8B). Our research demonstrated that hypoxia-induced EndMT of HCMECs activated Notch signaling and RUNX3 is likely to be the downstream target of Notch signaling pathway.

\section{Discussion}

Endothelial cells play an important role in cardiac functions. Previous research has shown that disorders in endothelial function are associated with adverse cardiac remodeling $(12,21)$. 
A

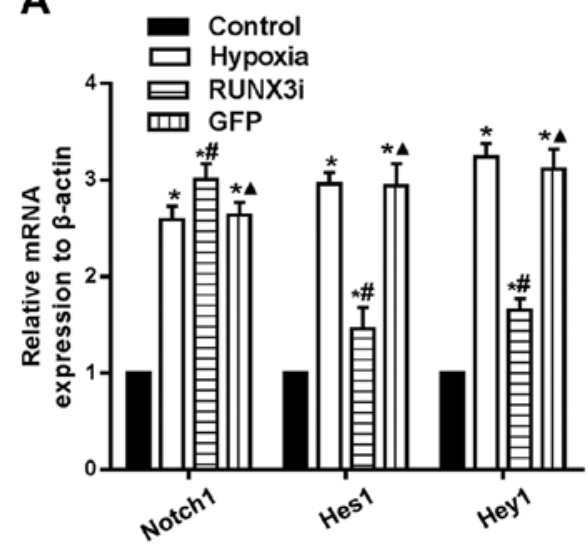

B

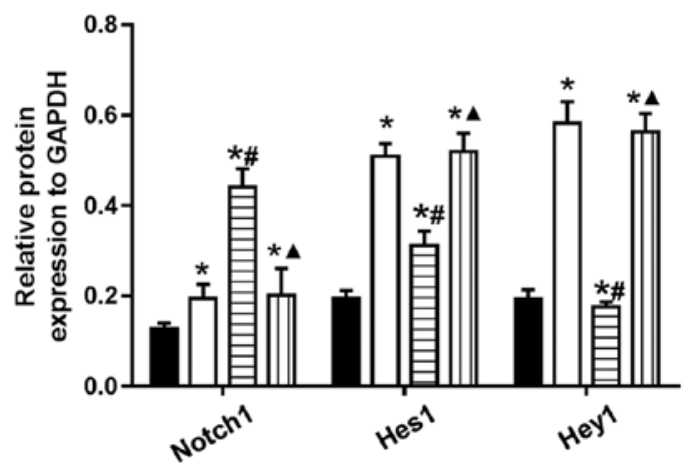

Figure 8. Changes in Notch signaling in hypoxia-induced endothelial-mesenchymal transition (EndMT). (A) mRNA levels of Notch1, Hes1 and Hey1 in human cardiac microvascular endothelial cells (HCMECs) were assessed by RT-PCR and normalized to reference gene $\beta$-actin ( $\mathrm{n}=7$, ${ }^{*} \mathrm{P}<0.05$ vs. control group; ${ }^{*} \mathrm{P}<0.05$ vs. hypoxia group; ${ }^{\wedge} \mathrm{P}<0.05$ vs. RUNX3i group). (B) Protein levels of Notch1, Hes1 and Hey1 in HCMECs was assessed by western blotting and normalized to that of GAPDH ( $\mathrm{n}=7,{ }^{*} \mathrm{P}<0.05$ vs. control group; ${ }^{*} \mathrm{P}<0.05$ vs. hypoxia group; ${ }^{\wedge} \mathrm{P}<0.05$ vs. RUNX3i group).

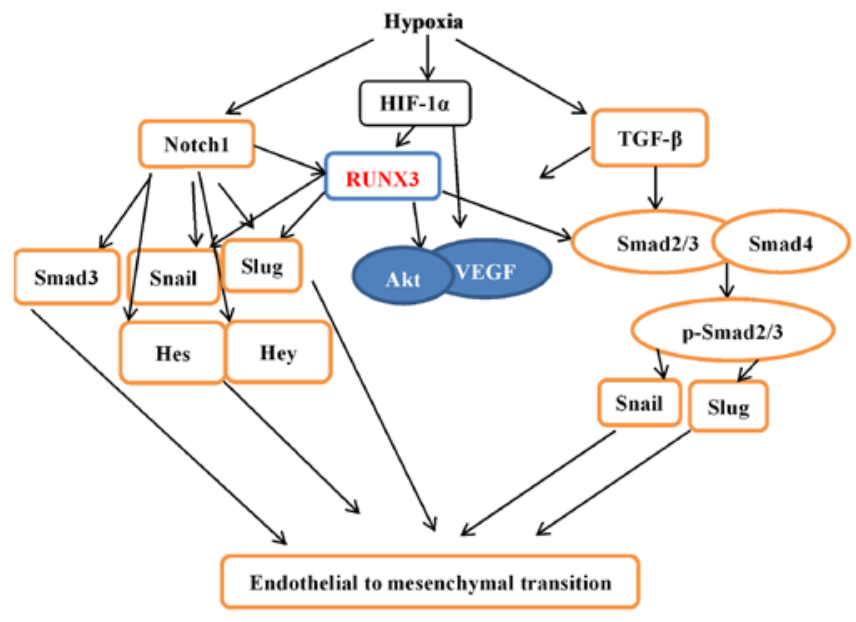

Figure 9. Crosstalk of hypoxia-induced endothelial-mesenchymal transition (EndMT) signaling pathways. Under hypoxic conditions, transcription factor hypoxia inducible factor-1 $\alpha$ (HIF-1 $\alpha)$ protein is accumulated and functionally activated, which induces the expression of genes associated with EndMT, such as Notch1, transforming growth factor- $\beta$ (TGF- $\beta$ ), and runtrelated transcription factor 3 (RUNX3), and the stabilization of Snail and Slug. RUNX3 is likely to be a direct target of HIF-1 $\alpha$, which can stabilize the Notch-ICD. The Notch-ICD also promotes the expression of EndMTassociated genes. Hypoxia can also activate the TGF- $\beta$ signaling pathway through $S$ mad2/3 proteins to induce EndMT transcription factor expression. $\mathrm{ICD}$, intracellular domain.

HCMECs can modulate vascular tone by releasing several endothelium-derived contracting and relaxing factors, by regulating and degradating vasoactive peptides, and through enzymes located on the endothelial surface. Therefore, they exert physiological and pathophysiological effects on the function of cardiac myocytes. In addition, HCMECs can grow rapidly in vitro with the cell number doubling in two to three days in culture, making the in vitro experiment feasible.

Various diseases, such as fibrosis and cancer, develop EndMT under hypoxia as a result of ischemic conditions. One previous study showed that the lack of oxygen inhibited prolyl hydroxylases, which are important in degrading HIF-1 $\alpha$ under normoxic conditions (23). Previous studies have also shown that HIF-1 $\alpha$ could induce the expression of EndMT-associated transcriptional factors, such as Snail, Slug and TGF- $\beta(20,24)$. In the present study, we demonstrated that hypoxia induced the transition of HCMECs to mesenchymal cells. However, the underlying molecular mechanism remains unknown.

In our study, we found that RUNX3 mRNA expression was low in HCMECs but increased when stimulated by hypoxia, which modulates intracellular signaling pathways and plays an important role in vascular endothelial cells. Several studies have demonstrated that RUNX3 is a downstream effector of TGF- $\beta$ and Notch signaling pathways, and plays critical roles in regulating cell functions, such as angiogenesis, cell migration and apoptosis $(14,15,17,25)$. Our results showed that TGF- $\beta$ and Notch signaling were activated during the hypoxia-induced EndMT of HCMECs. Knockdown of RUNX3 by RUNX3-RNAi lentivirus suppressed the mRNA and protein expression of mesenchymal markers and induced endothelial markers, suggesting that RUNX3 contributed to EndMT. Our research not only demonstrated that hypoxia induced EndMT in HCMECs but also provides a novel molecular mechanism (Fig. 9).

Several studies have demonstrated that cell migration ability is enhanced and angiogenesis is suppressed during EndMT $(2,17,26,27)$. However, our research showed that hypoxia markedly promoted tube formation and induced cell migration and that hypoxia stabilized HIF-1 $\alpha$ and induced the expression of VEGF. HIF-1 $\alpha$ also increases VEGF expression (28). Therefore, a possible explanation would be that these factors enhanced angiogenesis and cell migration, while EndMT partially offset the effects.

Recent research shows that hypoxia and the TGF- $\beta$ / Smad signaling pathways synergistically induce EndMT (20). TGF- $\beta$ and Notch signaling pathways were found to be the most common signaling mechanisms promoting EndMT participation in cardiovascular development and disease progression $(2,5,10,29,30)$. EndMT is a key point in cardiovascular development and disease. Our present study confirmed that the knockdown of RUNX3 attenuated EndMT of HCMECs, positively impacted the angiogenic phenotype, and reduced endothelial cell migration. The expression levels 
of TGF- $\beta 1$ and Notch1 were upregulated, while downstream genes were downregulated, suggesting that RUNX3 is a common downstream target of TGF- $\beta$ and Notch signaling pathways. Since our study was carried out in vitro, and the in vivo environment is more complex, further studies are warranted to gain a better understanding.

In conclusion, the present study demonstrated that RUNX3 plays an important role in endothelial cells. Our findings lay the foundation for further in vitro experiments. In addition, our study provided a novel molecular mechanism for CVD and further underscored the importance of RUNX3 during EndMT.

\section{Acknowledgements}

This study was supported by the National Natural Science Foundation of China (81041097 and 81460046) and the Natural Science Foundation of Jiangxi Province (20142BAB205040). We thank Dr Wan Zhang and Dr Junyi Zeng for expert technical assistance.

\section{References}

1. Chen W, Gao R, Liu L, Zhu M, Wang W, Wang Y, Wu Z, Li H, Zheng Z, Jiang $\mathrm{L}$ and $\mathrm{Hu} \mathrm{S}$ : Outline of the report on cardiovascular disease in China (2014). Chin Circ J 30: 617-622, 2015 (In Chinese).

2. Zeisberg EM, Tarnavski O, Zeisberg M, Dorfman AL, McMullen JR, Gustafsson E, Chandraker A, Yuan X, Pu WT, Roberts $\mathrm{AB}$, et al: Endothelial-to-mesenchymal transition contributes to cardiac fibrosis. Nat Med 13: 952-961, 2007.

3. Zeisberg EM and Kalluri R: Origins of cardiac fibroblasts. Circ Res 107: 1304-1312, 2010.

4. Oka T, Akazawa H, Naito AT and Komuro I: Angiogenesis and cardiac hypertrophy: maintenance of cardiac function and causative roles in heart failure. Circ Res 114: 565-571, 2014.

5. Kovacic JC, Mercader N, Torres M, Boehm M and Fuster V: Epithelial-to-mesenchymal and endothelial-to-mesenchymal transition: from cardiovascular development to disease. Circulation 125: 1795-1808, 2012.

6. Ranchoux B, Antigny F, Rucker-Martin C, Hautefort A, Péchoux C, Bogaard HJ, Dorfmüller P, Remy S, Lecerf F, Planté S, et al: Endothelial-to-mesenchymal transition in pulmonary hypertension. Circulation 131: 1006-1018, 2015.

7. Richards J, El-Hamamsy I, Chen S, Sarang Z, Sarathchandra P, Yacoub MH, Chester AH and Butcher JT: Side-specific endothelial-dependent regulation of aortic valve calcification: interplay of hemodynamics and nitric oxide signaling. Am J Pathol 182: 1922-1931, 2013.

8. Yao Y, Jumabay M, Ly A, Radparvar M, Cubberly MR and Boström KI: A role for the endothelium in vascular calcification. Circ Res 113: 495-504, 2013.

9. Hashimoto N, Phan SH, Imaizumi K, Matsuo M, Nakashima H, Kawabe T, Shimokata K and Hasegawa Y: Endothelial-mesenchymal transition in bleomycin-induced pulmonary fibrosis. Am J Respir Cell Mol Biol 43: 161-172, 2010.

10. Liu J, Dong F, Jeong J, Masuda T and Lobe CG: Constitutively active Notch1 signaling promotes endothelial mesenchymal transition in a conditional transgenic mouse model. Int J Mol Med 34: 669-676, 2014.

11. Aisagbonhi O, Rai M, Ryzhov S, Atria N, Feoktistov I and Hatzopoulos AK: Experimental myocardial infarction triggers canonical Wnt signaling and endothelial-to-mesenchymal transition. Dis Model Mech 4: 469-483, 2011.

12. Xu X, Tan X, Tampe B, Sanchez E, Zeisberg M and Zeisberg EM: Snail is a direct target of hypoxia-inducible factor $1 \alpha$ (HIF1 $\alpha)$ in hypoxia-induced endothelial to mesenchymal transition of human coronary endothelial cells. J Biol Chem 290: $16653-16664,2015$.
13. Peng Z, Wei D, Wang L, Tang H, Zhang J, Le X, Jia Z, Li Q and Xie K: RUNX3 inhibits the expression of vascular endothelial growth factor and reduces the angiogenesis, growth, and metastasis of human gastric cancer. Clin Cancer Res 12: 6386-6394, 2006.

14. Fu Y, Chang AC, Fournier M, Chang L, Niessen K and Karsan A: RUNX3 maintains the mesenchymal phenotype after termination of the Notch signal. J Biol Chem 286: 11803-11813, 2011.

15. Chen F, Liu X, Bai J, Pei D and Zheng J: The emerging role of RUNX3 in cancer metastasis (Review). Oncol Rep 35: 1227-1236, 2016.

16. Xu Q, Meng S, Liu B, Li MQ, Li Y, Fang L and Li YG: MicroRNA-130a regulates autophagy of endothelial progenitor cells through Runx3. Clin Exp Pharmacol Physiol 41: 351-357, 2014.

17. Meng S, Cao J, Zhang X, Fan Y, Fang L, Wang C, Lv Z, Fu D and Li Y: Downregulation of microRNA-130a contributes to endothelial progenitor cell dysfunction in diabetic patients via its target Runx3. PLoS One 8: e68611, 2013.

18. Zhang J, Li B, Zheng Z, Kang T, Zeng M, Liu Y and Xia B: Protective effects of Notch1 signaling activation against high glucose-induced myocardial cell injury: analysis of its mechanisms of action. Int J Mol Med 36: 897-903, 2015.

19. Ma FX, Zhou B, Chen Z, Ren Q, Lu SH, Sawamura T and Han ZC: Oxidized low density lipoprotein impairs endothelial progenitor cells by regulation of endothelial nitric oxide synthase. J Lipid Res 47: 1227-1237, 2006.

20. Xu X, Tan X, Hulshoff MS, Wilhelmi T, Zeisberg M and Zeisberg EM: Hypoxia-induced endothelial-mesenchymal transition is associated with RASAL1 promoter hypermethylation in human coronary endothelial cells. FEBS Lett 590: 1222-1233, 2016.

21. Lee SW, Won JY, Kim WJ, Lee J, Kim KH, Youn SW, Kim JY, Lee EJ, Kim YJ, Kim KW, et al: Snail as a potential target molecule in cardiac fibrosis: paracrine action of endothelial cells on fibroblasts through snail and CTGF axis. Mol Ther 21: 1767-1777, 2013.

22. Frías A, Lambies G, Viñas-Castells R, Martínez-Guillamon C, Dave N, García de Herreros A and Díaz VM: A switch in Akt isoforms is required for Notch-induced Snaill expression and protection from cell death. Mol Cell Biol 36: 923-940, 2015.

23. Gonzalez DM and Medici D: Signaling mechanisms of the epithelial-mesenchymal transition. Sci Signal 7: re8, 2014.

24. Watson CJ, Collier P, Tea I, Neary R, Watson JA, Robinson C, Phelan D, Ledwidge MT, McDonald KM, McCann A, et al: Hypoxia-induced epigenetic modifications are associated with cardiac tissue fibrosis and the development of a myofibroblast-like phenotype. Hum Mol Genet 23: 2176-2188, 2014.

25. Zheng Z, Zhu L, Zhang X, Li L, Moon S, Roh MR and Jin Z: RUNX3 expression is associated with sensitivity to pheophorbide a-based photodynamic therapy in keloids. Lasers Med Sci 30: $67-75,2015$.

26. Tang RN, Lv LL, Zhang JD, Dai HY, Li Q, Zheng M, Ni J, Ma KL and Liu BC: Effects of angiotensin II receptor blocker on myocardial endothelial-to-mesenchymal transition in diabetic rats. Int J Cardiol 162: 92-99, 2013.

27. Zhou X, Chen X, Cai JJ, Chen LZ, Gong YS, Wang LX, Gao Z, Zhang HQ, Huang WJ and Zhou H: Relaxin inhibits cardiac fibrosis and endothelial-mesenchymal transition via the Notch pathway. Drug Des Devel Ther 9: 4599-4611, 2015.

28. Vasconcelos RC, Costa AL, Freitas RA, Bezerra BA, Santos BR, Pinto LP and Gurgel BC: Immunoexpression of HIF- $1 \alpha$ and VEGF in periodontal disease and healthy gingival tissues. Braz Dent J 27: 117-122, 2016.

29. Garside VC, Chang AC, Karsan A and Hoodless PA: Co-ordinating Notch, BMP, and TGF- $\beta$ signaling during heart valve development. Cell Mol Life Sci 70: 2899-2917, 2013.

30. Niessen K and Karsan A: Notch signaling in cardiac development. Circ Res 102: 1169-1181, 2008. 